\title{
TROPICAL CRYPTOGRAPHY
}

\author{
DIMA GRIGORIEV AND VLADIMIR SHPILRAIN
}

\begin{abstract}
We employ tropical algebras as platforms for several cryptographic schemes that would be vulnerable to linear algebra attacks were they based on "usual" algebras as platforms.
\end{abstract}

Keywords: tropical algebra, public key exchange, encryption

Mathematics subject classification: 15A80, 94A60.

\section{INTRODUCTION}

In this paper, we employ tropical algebras as platforms for several cryptographic schemes. The schemes themselves are not brand new; similar ideas were used in the "classical" case, i.e., for algebras with the familiar addition and multiplication. However, in the classical case these schemes were shown to be vulnerable to various linear algebra attacks. Here we make a case for using tropical algebras as platforms by using, among other things, the fact that in the "tropical" case, even solving systems of linear equations is computationally infeasible in general. Yet another advantage is improved efficiency because in tropical schemes, one does not have to perform any multiplications of numbers since tropical multiplication is the usual addition, see below.

We start by giving some necessary information on tropical algebras here; for more details, we refer the reader to a recent monograph [2].

Consider a tropical semiring $S$ (also known as the min-plus algebra due to the following definition). This semiring is defined as a subset of reals that contains 0 and is closed under addition, with two operations as follows:

$x \oplus y=\min (x, y)$

$x \otimes y=x+y$.

It is straightforward to see that these operations satisfy the following properties:

associativity:

$x \oplus(y \oplus z)=(x \oplus y) \oplus z$

$x \otimes(y \otimes z)=(x \otimes y) \otimes z$.

Research of the first author was partially supported by the Federal Agency of the Science and Innovations of Russia, State Contract No. 02.740.11.5192.

Research of the second author was partially supported by the NSF grants DMS-0914778 and CNS1117675 . 
commutativity:

$x \oplus y=y \oplus x$

$x \otimes y=y \otimes x$.

distributivity:

$(x \oplus y) \otimes z=(x \otimes z) \oplus(y \otimes z)$.

There are some "counterintuitive" properties as well:

$x \oplus x=x$

$x \otimes 0=x$

$x \oplus 0$ could be either 0 or $x$.

There is also a special " $\epsilon$-element" $\epsilon=\infty$ such that, for any $x \in S$,

$\epsilon \oplus x=x$

$\epsilon \otimes x=\epsilon$.

A (tropical) monomial in $S$ looks like a usual linear function, and a tropical polynomial is the minimum of a finite number of such functions, and therefore a concave, piecewise linear function. The rules for the order in which tropical operations are performed are the same as in the classical case, see the example below.

Example 1. Here is an example of a tropical monomial: $x \otimes x \otimes y \otimes z \otimes z$. The (tropical) degree of this monomial is 5 . We note that sometimes, people use the alternative notation $x^{\otimes 2}$ for $x \otimes x$, etc.

An example of a tropical polynomial is: $p(x, y, z)=5 \otimes x \otimes y \otimes z \oplus x \otimes x \oplus 2 \otimes z \oplus 17=$ $(5 \otimes x \otimes y \otimes z) \oplus(x \otimes x) \oplus(2 \otimes z) \oplus 17$. This polynomial has (tropical) degree 3, by the highest degree of its monomials.

We note that, just as in the classical case, a tropical polynomial is canonically represented by an ordered set of tropical monomials (together with non-zero coefficients), where the order that we use here is deglex.

While the $\oplus$ operation is obviously not invertible, the $\otimes$ operation is, and we denote the inverse of this operation by $\oslash$ (it is just the classical subtraction):

$x \oslash y=z$ if and only if $y \otimes z=x$.

We refer to 8 for more detailed properties of this operation; here we just mention the following properties that agree with those of the usual division:

$(x \oslash y) \otimes(z \oslash t)=(x \otimes z) \oslash(y \otimes t)$

$(x \oslash y) \oplus(z \oslash t)=((x \otimes t) \oplus(y \otimes z)) \oslash(y \otimes t)$.

Also as in the classical case, there is an equivalence relation on the set of all expressions of the form $x \oslash y$ :

$x \oslash y$ is equivalent to $z \oslash t$ if and only if $x \otimes t=y \otimes z$.

All expressions of the form $x \oslash y$, where $x, y \in S$, modulo the above equivalence, form a semifield (of quotients of $S$ ), which we denote by $\operatorname{Rat}(S)$, see [8]. 
1.1. Tropical matrix algebra. A tropical algebra can be used for matrix operations as well. To perform the $A \oplus B$ operation, the elements $m_{i j}$ of the resulting matrix $M$ are set to be equal to $a_{i j} \oplus b_{i j}$. The $\otimes$ operation is similar to the usual matrix multiplication, however, every "+" calculation has to be substituted by a $\oplus$ operation, and every "." calculation by a $\otimes$ operation.

Example 2. $\left(\begin{array}{cc}1 & 2 \\ 5 & -1\end{array}\right) \oplus\left(\begin{array}{ll}0 & 3 \\ 2 & 8\end{array}\right)=\left(\begin{array}{cc}0 & 2 \\ 2 & -1\end{array}\right)$.

Example 3. $\left(\begin{array}{cc}1 & 2 \\ 5 & -1\end{array}\right) \otimes\left(\begin{array}{ll}0 & 3 \\ 2 & 8\end{array}\right)=\left(\begin{array}{ll}1 & 4 \\ 1 & 7\end{array}\right)$.

The role of the identity matrix $I$ is played by the matrix that has " 0 " $s$ on the diagonal and $\infty$ elsewhere. Similarly, a scalar matrix would be a matrix with an element $\lambda \in S$ on the diagonal and $\infty$ elsewhere. Such a matrix commutes with any other square matrix (of the same size). Multiplying a square matrix by a scalar amounts to multiplying it by the corresponding scalar matrix.

Example 4. $2 \otimes\left(\begin{array}{cc}1 & 2 \\ 5 & -1\end{array}\right)=\left(\begin{array}{cc}2 & \infty \\ \infty & 2\end{array}\right) \otimes\left(\begin{array}{cc}1 & 2 \\ 5 & -1\end{array}\right)=\left(\begin{array}{ll}3 & 4 \\ 7 & 1\end{array}\right)$.

Then, tropical diagonal matrices have something on the diagonal and $\infty$ elsewhere.

We also note that, in contrast with the "classical" situation, it is rather rare that a "tropical" matrix is invertible. More specifically (see [2, p.5]), the only invertible tropical matrices are those that are obtained from a diagonal matrix by permuting rows and/or columns.

\section{Key EXChANGe USING MATRICES OVER A TROPICAL ALGEBRA}

We are now going to offer a key exchange protocol building on an idea of Stickel [13] who used it for matrices over "usual" algebras, which made his scheme vulnerable to linear algebra attacks, see e.g. [11]. Since we believe that Stickel's idea itself has a good potential, we suggest here to use matrices over a tropical algebra as the platform for his scheme, in order to prevent linear algebra attacks.

We start by recalling the original Stickel's protocol. Let $G$ be a public noncommutative semigroup, $a, b \in G$ public elements such that $a b \neq b a$. The key exchange protocol goes as follows.

\subsection{Protocol 1 [13].}

(1) Alice picks two random natural numbers $n, m$ and sends $u=a^{n} b^{m}$ to Bob.

(2) Bob picks two random natural numbers $r, s$ and sends $v=a^{r} b^{s}$ to Alice.

(3) Alice computes $K_{A}=a^{n} v b^{m}=a^{n+r} b^{m+s}$.

(4) Bob computes $K_{B}=a^{r} u b^{s}=a^{n+r} b^{m+s}$.

Thus, Alice and Bob end up with the same group element $K=K_{A}=K_{B}$ which can serve as the shared secret key.

This can be generalized if the platform is not just a semigroup, but a ring (actually, a semiring would suffice): 
2.2. Protocol 2 [6, 11]. Let $R$ be a public non-commutative ring (or a semiring), $a, b \in R$ public elements such that $a b \neq b a$.

(1) Alice picks two random polynomials $p_{1}(x), p_{2}(x)$ (say, with positive integer coefficients) and sends $p_{1}(a) \cdot p_{2}(b)$ to Bob.

(2) Bob picks two random polynomials $q_{1}(x), q_{2}(x)$ and sends $q_{1}(a) \cdot q_{2}(b)$ to Alice.

(3) Alice computes $K_{A}=p_{1}(a) \cdot\left(q_{1}(a) \cdot q_{2}(b)\right) \cdot p_{2}(b)$.

(4) Bob computes $K_{B}=q_{1}(a) \cdot\left(p_{1}(a) \cdot p_{2}(b)\right) \cdot q_{2}(b)$.

Thus, since $p_{1}(a) \cdot q_{1}(a)=q_{1}(a) \cdot p_{1}(a)$ and $p_{2}(b) \cdot q_{2}(b)=q_{2}(b) \cdot p_{2}(b)$, Alice and Bob end up with the same element $K=K_{A}=K_{B}$ which can serve as the shared secret key.

It is Protocol 2 that we propose to adopt in the "tropical" situation.

2.3. Protocol 3 (tropical). Let $R$ be the tropical algebra of $n \times n$ matrices over integers, and let $A, B \in R$ be public matrices such that $A \otimes B \neq B \otimes A$.

(1) Alice picks two random tropical polynomials $p_{1}(x), p_{2}(x)$ (with integer coefficients) and sends $p_{1}(A) \otimes p_{2}(B)$ to Bob.

(2) Bob picks two random tropical polynomials $q_{1}(x), q_{2}(x)$ and sends $q_{1}(A) \otimes q_{2}(B)$ to Alice.

(3) Alice computes $K_{A}=p_{1}(A) \otimes\left(q_{1}(A) \otimes q_{2}(B)\right) \otimes p_{2}(B)$.

(4) Bob computes $K_{B}=q_{1}(A) \otimes\left(p_{1}(A) \otimes p_{2}(B)\right) \otimes q_{2}(B)$.

Thus, since $p_{1}(A) \otimes q_{1}(A)=q_{1}(A) \otimes p_{1}(A)$ and $p_{2}(B) \otimes q_{2}(B)=q_{2}(B) \otimes p_{2}(B)$, Alice and Bob end up with the same element $K=K_{A}=K_{B}$ which can serve as the shared secret key.

2.4. What are the advantages of the "tropical" Protocol 3 over "classical" Protocols 1 and 2? One obvious advantage is improved efficiency because when multiplying matrices in the tropical sense, one does not have to perform any multiplications of numbers since tropical multiplication is the "usual" addition.

To compare security, we briefly recall a linear algebra attack [11] on Stickel's original protocol (Protocol 1), where $G$ was a group of invertible matrices over a field. In that case, to recover a shared key $K$, it is not necessary to find the exponents $n, m, r$, or $s$. Instead, as was shown in [11, it is sufficient for the adversary to find matrices $x$ and $y$ such that $x a=a x, y b=b y$, and $x u=y$. (Here $x$ corresponds to $a^{-n}$, while $y$ corresponds to $b^{m}$.)

These conditions translate into a system of $3 k^{2}$ linear equations with $2 k^{2}$ unknowns, where $k$ is the size of the matrices. This typically yields a unique solution (according to computer experiments of [11] and [10]), which can be efficiently found if the matrices are considered over a field.

We note that in [10, a more sophisticated attack on a more general Protocol 2 was offered. This attack applies to not necessarily invertible matrices over a field.

In the "tropical" situation (Protocol 3), however, a linear algebra attack will not work, for several reasons: 
(1) Matrices are generically not invertible, so the equation $X Y=U$ with known $U$ and unknown $X, Y$ does not translate into a system of linear equations.

(2) The equations $X A=A X, Y B=B Y$ do translate into a system of linear equations, which may be called a "two-sided min-linear system", following [2]. In [1, it is shown that the problem of solving such systems is in the class $\mathbf{N P} \cap \mathbf{C o}-\mathbf{N P}$ (there is a belief that it does not belong to the class $\mathbf{P}$ ). We refer to [2] for a comprehensive exposition of what is known concerning existing algorithms for solving two-sided min-linear systems and their complexity. Here we just say that, while it is known how to find one of the solutions of a system (if a solution exists), there is no known efficient method for describing the linear space of all solutions, in contrast with the "classical" situation.

2.5. Parameters and key generation. Here we suggest values of the parameters involved in the description of our Protocol 3.

- The size of matrices $n=10$.

- The entries of the public matrices $A, B$ are integers, selected uniformly randomly in the range $\left[-10^{10}, 10^{10}\right]$.

- The degrees of the tropical polynomials $p_{1}(x), p_{2}(x), q_{1}(x), q_{2}(x)$ are selected uniformly randomly in the range $[1,10]$.

- The coefficients of the above tropical polynomials are selected uniformly randomly in the range $[-1000,1000]$.

With these parameters, the size of the key space (for private tropical polynomials) is approximately $10^{30}$.

\section{ENCRYPTION USING BIRATIONAL AUTOMORPHISMS OF A TROPICAL POLYNOMIAL}

\section{ALGEBRA}

In this section, we describe a public key encryption scheme that would be susceptible to a linear algebra attack in the "classical" case (cf. [9], 44), but not in the "tropical" case.

Let $P=\operatorname{Rat}\left[x_{1}, \ldots, x_{n}\right]$ be the quotient semifield of a tropical polynomial algebra over $\mathbf{Z}$.

3.1. The protocol. There is a public automorphism $\alpha \in \operatorname{Aut}(P)$ given as a tuple of tropical rational functions $\left(\alpha\left(x_{1}\right), \ldots, \alpha\left(x_{n}\right)\right)$. Alice's private key is $\alpha^{-1}$. Note that $\alpha$ is also a bijection of the set $\mathbf{Z}^{n}$, i.e., it is a one-to-one map of the set of all $n$-tuples of integers onto itself. We will use the same notation $\alpha$ for an automorphism of $P$ and for the corresponding bijection of $\mathbf{Z}^{n}$, hoping this will not cause a confusion.

(1) Bob's secret message is a tuple of integers $s=\left(s_{1}, \ldots, s_{n}\right) \in \mathbf{Z}^{n}$. Bob encrypts his tuple by applying the public automorphism $\alpha: E_{\alpha}(s)=\alpha\left(s_{1}, \ldots, s_{n}\right)$.

(2) Alice decrypts by applying her private $\alpha^{-1}$ to the tuple $E_{\alpha}(s): \alpha^{-1}\left(E_{\alpha}(s)\right)=$ $s=\left(s_{1}, \ldots, s_{n}\right)$. 
3.2. Key generation. The crucial ingredient in this scheme is, of course, generating the public key $\alpha \in A u t(P)$. Alice can generate her automorphism $\alpha$ as a product of "monomial" automorphisms on the set of variables $\left\{x_{1}, \ldots, x_{n}\right\}$ and "triangular" automorphisms of the form

$$
\varphi: x_{i} \rightarrow x_{i} \otimes p_{i}\left(x_{i+1}, \ldots, x_{n}\right), 1 \leq i \leq n,
$$

where $p_{i} \in P=\operatorname{Rat}\left[x_{1}, \ldots, x_{n}\right]$. Each triangular automorphism, in turn, is a product of "elementary" triangular automorphisms; these are of the form

$$
\tau: x_{j} \rightarrow x_{j} \otimes q_{j}\left(x_{j+1}, \ldots, x_{n}\right), x_{k} \rightarrow x_{k}, k \neq j .
$$

The inverse of such a $\tau$ is

$$
\tau^{-1}: x_{j} \rightarrow x_{j} \oslash\left(q_{j}\left(x_{j+1}, \ldots, x_{n}\right)\right), x_{k} \rightarrow x_{k}, k \neq j,
$$

where $q_{j} \in P$.

"Monomial" automorphisms are analogs of linear automorphisms in the "classical" situation; they are of the form

$$
\mu: x_{i} \rightarrow b_{i} \otimes x_{1}^{\otimes a_{i 1}} \otimes \cdots \otimes x_{n}^{\otimes a_{i n}},
$$

where $b_{i}$ are finite coefficients (i.e., $b_{i} \neq \infty$ ), and the matrix $A=\left(a_{i j}\right)$ of integer exponents is invertible in the "classical" sense.

We note, in passing, that a question of independent interest (independent of cryptographic applications) is:

Problem 1. Is every automorphism of $P=\operatorname{Rat}\left[x_{1}, \ldots, x_{n}\right]$, the quotient semifield of a tropical polynomial algebra over $\mathbf{Z}$, a product of triangular and monomial automorphisms?

3.3. Parameters. We suggest the following parameters.

- The number $n$ of variables in the platform tropical polynomial algebra: 10.

- The number of triangular automorphisms in a product for $\alpha: 2$. The number of monomial automorphisms: 3. More specifically, Alice generates her $\alpha$ in the following form:

$$
\alpha=\mu_{1} \circ \varphi_{1} \circ \mu_{2} \circ \varphi_{2} \circ \mu_{3},
$$

where $\varphi_{1}, \varphi_{2}$ are triangular automorphisms, and $\mu_{1}, \mu_{2}, \mu_{3}$ are monomial automorphisms.

- The tropical degrees of all $q_{j}$ are equal to 2 .

- The coefficients of the above tropical polynomials $q_{j}$ are selected uniformly randomly in the range $[-10,10]$.

Remark 1. Alice can obtain the inverse of $\alpha$ as the product of inverses of the automorphisms $\varphi_{i}$ and $\mu_{i}$, in the reverse order. However, Alice does not have to compute an explicit expression for $\alpha^{-1}$; this computation may not be efficient since the degree of $\alpha^{-1}$ may be substantially greater than the degree of $\alpha$. In our protocol, Alice has to 
apply $\alpha^{-1}$ to a particular point in $\mathbf{Z}^{n}$; efficient way of doing this is to first apply $\mu_{3}^{-1}$, then apply $\varphi_{2}^{-1}$ to the obtained point, etc.

Remark 2. There is a ramification of the above protocol, where Bob's secret message is a tropical polynomial $u$, instead of a point in $\mathbf{Z}^{n}$. (Note that the result of encrypting $u$ will be, in general, an element of $P=\operatorname{Rat}\left[x_{1}, \ldots, x_{n}\right]$.) In this ramification, decryption is going to have a much higher computational complexity because Alice would have to compute an explicit expression for $\alpha^{-1}$ (cf. the previous remark). On the other hand, encryption in this case is going to be homomorphic (in the "tropical" sense) because $\alpha\left(u_{1} \oplus u_{2}\right)=\alpha\left(u_{1}\right) \oplus \alpha\left(u_{2}\right)$ and $\alpha\left(u_{1} \otimes u_{2}\right)=\alpha\left(u_{1}\right) \otimes \alpha\left(u_{2}\right)$. For examples of homomorphic encryption in the "classical" case see e.g. [5] or [7].

Remark 3. One can consider an encryption protocol, similar to the one above, also in the "classical" case. As we have already pointed out, polynomial automorphisms were employed in a similar context in [9], but birational automorphisms have not been used for cryptographic purposes before, to the best of our knowledge.

3.4. Possible attacks. There are the following two attacks that adversary may attempt.

(1) Trying to compute $\alpha^{-1}$ from the public automorphism $\alpha$. The problem with this attack is that the degree of $\alpha^{-1}$ may be exponentially greater than the degree of $\alpha$, which makes any commonly used attack (e.g. a linear algebra attack) infeasible.

(2) Trying to recover Bob's secret message $s$ from $\alpha(s)$. This translates into a system of tropical polynomial equations; solving such a system is an NP-hard problem, as we show in the following proposition.

Proposition 1. The problem of solving systems of tropical polynomial equations is NP-hard.

Before getting to the proof, we note that for a closely related, but different, problem of emptiness of a tropical variety NP-completeness was established in [14].

Proof. We show how to reduce the SAT problem to the problem of solving a system of tropical polynomial equations. Recall that the SAT (for SATisfiability) problem is a decision problem, whose instance is a Boolean expression written using only AND, OR, NOT, variables, and parentheses. The question is: given the expression, is there some assignment of TRUE $(=1)$ and FALSE $(=0)$ values to the variables that will make the entire expression true? A formula of propositional logic is said to be satisfiable if logical values can be assigned to its variables in a way that makes the formula true. The Boolean satisfiability problem is NP-complete [3]. The problem remains NP-complete even if all expressions are written in conjunctive normal form with 3 variables per clause (3-CNF), yielding the 3-SAT problem.

Suppose now we have a 3-CNF, and we are going to build (in time polynomial in the number of clauses) a system of tropical polynomial equations that has a solution if and only if the given 3-CNF is satisfiable. Denote Boolean variables in the given 3 -CNF by $u_{i}$. In our tropical system, we are going to have two kinds of variables: those 
corresponding to literals $u_{i}$ will be denoted by $x_{i}$, and those corresponding to literals $\neg u_{i}$ will be denoted by $y_{i}$.

First of all, we include in our tropical system all equations of the form $x_{i} \otimes y_{i}=1$, for all $i$.

Now suppose we have a clause with 3 literals, for example, $u_{i} \vee \neg u_{j} \vee \neg u_{k}$. To this clause, we correspond the following tropical polynomial equation:

$$
y_{i} \oplus x_{j} \oplus x_{k}=0 .
$$

Obviously, the above clause is TRUE if and only if either $u_{i}=1$, or $u_{j}=0$, or $u_{k}=0$. If $u_{i}=1$, then $y_{i}=0$, and our tropical equation is satisfied. If, say, $u_{j}=1$, then $x_{j}=0$, and again our tropical equation is satisfied. This shows that if a given 3 -CNF is satisfiable, then our tropical equation has a solution.

If, on the other hand, our tropical equation has a solution, that means either $y_{i}=0$, or $x_{j}=0$, or $x_{k}=0$. In any case, the given clause is easily seen to be TRUE upon corresponding $u_{i}$ to $x_{i}$ and $\neg u_{i}$ to $y_{i}$. (Note that if, say, $y_{i}=0$, then, since we also have the equation $x_{i} \otimes y_{i}=1, x_{i}$ should be equal to 1 .)

Having thus built a tropical equation for each clause in the given 3-CNF, we end up with a system of tropical polynomial equations that corresponds to the whole 3-CNF, which is solvable if and only if the given $3-\mathrm{CNF}$ is satisfiable. This completes the proof.

Acknowledgement. Both authors are grateful to Max Planck Institut für Mathematik, Bonn for its hospitality during the work on this paper.

\section{REFERENCES}

[1] M. Bezem, R. Nieuwenhuis, E. Rodrguez-Carbonell, Hard problems in maxalgebra, control theory, hypergraphs and other areas, Information Processing Letters 110(4) (2010), 133-138.

[2] P. Butkovic, Max-linear systems: theory and algorithms, Springer-Verlag London, 2010.

[3] M. Garey, J. Johnson, Computers and Intractability, A Guide to NP-Completeness, W. H. Freeman, 1979.

[4] L. Goubin, N. Courtois, Cryptanalysis of the TTM cryptosystem, in: ASIACRYPT 2000, Lecture Notes in Comput. Sci. 1976 (2000), 44-57.

[5] D. Grigoriev, I. Ponomarenko, Constructions in public-key cryptography over matrix groups, Contemp. Math., Amer. Math. Soc. 418 (2006), 103-119.

[6] G. Maze, C. Monico, J. Rosenthal, Public key cryptography based on semigroup actions, Advances in Mathematics of Communications 4 (2007), 489-507.

[7] A. Menezes, P. van Oorschot, and S. Vanstone, Handbook of Applied Cryptography, CRC-Press 1996.

[8] G. Mikhalkin, Tropical geometry, in preparation. http://www.math.toronto.edu/mikha/book.pdf

[9] T. Moh, A public key system with signature and master key functions, Comm. Algebra 27 (1999), 2207-2222.

[10] C. Mullan, Cryptanalysing variants of Stickel's key agreement scheme, preprint.

[11] V. Shpilrain, Cryptanalysis of Stickel's key exchange scheme, in: Computer Science in Russia 2008, Lecture Notes Comp. Sc. 5010 (2008), 283-288. 
[12] R. Steinwandt and A. Suárez Corona, Cryptanalysis of a 2-party key establishment based on a semigroup action problem, Advances in Mathematics of Communications 5 (2011), 87-92.

[13] E. Stickel, A New Method for Exchanging Secret Keys. In: Proc. of the Third International Conference on Information Technology and Applications (ICITA05) 2 (2005), 426-430.

[14] T. Theobald, On the frontiers of polynomial computations in tropical geometry, J. Symbolic Comput. 41 (2006), 1360-1375.

CNRS, Mathématiques, Université de Lille, 59655, Villeneuve d'Ascq, France

E-mail address: dmitry.grigoryev@math.univ-lille1.fr

Department of Mathematics, The City College of New York, New York, NY 10031

E-mail address: shpil@groups.sci.ccny.cuny.edu 